\title{
Proteção em Redes Ópticas Elásticas com Multiplexação Espacial
}

\author{
Helder M. N. da S. Oliveira ${ }^{1}$, Nelson L. S. da Fonseca (orientador) ${ }^{1}$ \\ ${ }^{1}$ Universidade Estadual de Campinas (UNICAMP), Campinas, SP, Brasil \\ helder@lrc.ic.unicamp.br, nfonsecalic.unicamp.br
}

\begin{abstract}
Resumo. Este artigo resume as contribuições da Tese de doutorado intitulada "Proteção em Redes Ópticas Elásticas com Multiplexação Espacial". $\mathrm{Na}$ tese, propõem-se soluções de proteção para redes ópticas elásticas com multiplexação espacial, visando a redução do bloqueio de requisições para estabelecimento de conexões, bem como a redução da utilização dos recursos. Considerou-se a utilização de diferentes esquemas para proteção de caminhos, modulação adaptativa, agregação de tráfego, sobreposição de espectro em caminhos de proteção, roteamento com interferência mínima e roteamento por multicaminhos. Os algoritmos propostos permitirão a provisão de proteção em redes que transportarão um volume de tráfego muito maior do que os das redes ópticas atuais.
\end{abstract}

\begin{abstract}
In this paper, we summarize the contributions of the Doctoral Thesis entitled "Protection in Elastic Optical Networks with Spatial Multiplexing". This thesis proposes solutions for protection of elastic optical networks with spatial multiplexing, aiming at reducing the blocking of requests and the improvement of resource utilization. For this, we take into account the use of different schemes to paths protection, adaptive modulation, traffic grooming, spectrum overlap in protection paths, minimum interference routing and multipath routing. The algorithms proposed will allow the provisioning of protection in optical networks that will transport much greater volumes of traffic than do the current networks.
\end{abstract}

\section{Introdução}

Nas últimas décadas, o tráfego na Internet tem crescido exponencialmente, e não existe nenhuma indicação de que esta tendência cessará nas próximas décadas. Estima-se que, nos próximos dez anos, a taxa de acesso do usuário final irá crescer de $100 \mathrm{Mb} / \mathrm{s}$ para 1 $\mathrm{Gb} / \mathrm{s}$, como consequência das novas tecnologias de redes de acesso, em especial as redes 5G. Por outro lado, a camada de enlace de dados do núcleo da Internet é composta majoritariamente por redes ópticas com tecnologia de multiplexação por divisão de comprimento de onda (WDM). A capacidade (banda passante) disponível com emprego dessa tecnologia não será suficiente para atender às futuras demandas. Além disso, em redes WDM, a alocação de banda passante é feitas em unidade fixa correspondente a banda passante de um comprimento de onda, o que não favorece o oferecimento de serviços com diversidade de requisitos de banda passante.

A tecnologia de redes ópticas elástica (EON) com multiplexação espacial (SDM) sobre fibra multi-núcleo (MCF) é uma solução promissora para lidar com a crescente demanda de banda passante. Além disso, a possibilidade de se alocar flexivelmente o espectro permitirá que estas redes lidem com demandas de tráfego com requisitos de banda 
diversificados. No entanto, as EON-SDMs disponibilizarão maiores volumes de banda que as atuais redes com apenas um núcleo. Assim, sendo, há uma maior necessidade de proteção, uma vez que um corte de fibra ou falha de componentes de rede implicará em grandes perdas de dados, muito maiores do que nas atuais redes ópticas.

Um dos problemas fundamentais em EON é o problema de roteamento e alocação de espectro (RSA), cuja solução deve considerar a alocação contígua e contínua do espectro para todos os enlaces de um caminho óptico. Em EON-SDM, o RSA é estendido, pois se deve, também, alocar espectro em diferentes núcleos, sendo este problema mais complexo e designado por roteamento e alocação de núcleo e espectro (RSCA). Vários algoritmos de RSCA para EON-SDM foram propostos, recentemente, na literatura [Yang et al. 2018], [Yin et al. 2017], [Tode e Hirota 2017] e [Fujii et al. 2017]. Entretanto, apenas em [Tan et al. 2016] aborda-se a questão de proteção de caminhos.

Este artigo resume o trabalho da Tese "Proteção em Redes Ópticas Elásticas com Multiplexação Espacial" [Oliveira 2018], que investiga o problema de proteção em EONSDM com diferentes mecanismos de gerenciamento do espectro, tais como agregação de tráfego (traffic grooming), sobreposição de espectro (spectrum overlap) e modulação adaptativa (adaptive modulation). Foram propostos e avaliados 16 novos algoritmos para proteção de EON-SDM empregando diferentes mecanismos de proteção e gerenciamento de espectro, a saber:

- Três novos algoritmos considerando diferentes esquemas de proteção, tais como: compartilhamento de caminho de backup, $p$-cyle FIPP e $p$-cycle FIPP com interferência mínima;

- Três novos algoritmos que empregam modulação adaptativa. Os algoritmos propostos reduzem a utilização de recursos evitando a degradação da qualidade de transmissão no estabelecimento de novas conexões;

- Quatro novos algoritmos para redes que empregam sobreposição de espectro e agregação de tráfego;

- Três novos algoritmos para redes que empregam conjuntamente as técnicas de proteção fim-a-fim, modulação adaptativa, agregação de tráfego e sobreposição de espectro. A modulação adaptativa possibilita o envio de uma maior quantidade de bits por símbolos. A agregação de tráfego possibilita a não utilização de banda de guarda entre caminhos e a sobreposição de espectro reduz os recursos utilizados para proteção;

- Três novos algoritmos que empregam roteamento multicaminho para proteção de caminho, que reduzem a fragmentação de espectro gerada pela alocação e desalocação de caminhos em EON-SDM.

As contribuições da Tese avançam o estado da arte no projeto de EON-SDM e em proteção de redes ópticas. A relevância dessas contribuições é, portanto, possibilitar a evolução da tecnologia de EON-SDM, o que permitirá o aumento ainda maior da capacidade de transmissão da Internet e dotar a Internet de robustez, permitindo novas aplicações com grandes demandas de banda passante.

\section{Trabalhos Relacionados}

Trabalhos recentes abordam o estudo de roteamento e alocação de núcleo e espectro. Em [Yin et al. 2017] investiga-se a otimização de alocação cientes de crosstalk. Em [Yang et al. 2018] foram propostas uma solução baseada em programação linear inteira e uma heurística para maximizar a utilização do espectro. Em [Tode e Hirota 2017], 
considera-se a utilização de roteamento e alocação de modo e espectro. Em [Fujii et al. 2017], propõe-se uma arquitetura de nós AoD para tornar as EON-SDM energeticamente mais eficiente.

$\mathrm{Na}$ literatura, diversos trabalhos estudam proteção em redes ópticas. No entanto, dentre todos os trabalhados encontrados, apenas [Tan et al. 2016] trata de proteção de EON-SDM. [Tan et al. 2016] propõe o algoritmo Cap-DPP que utiliza caminhos dedicados para fornecer proteção de caminho, e utiliza uma técnica de k-caminhos para encontrar o caminho primário e o caminho de backup em EON-SDM. No entanto, o algoritmo Cap-DPP produz alocação ineficiente de espectro quando comparado aos algoritmos propostos em [Oliveira 2018].

\section{Algoritmos Desenvolvidos}

Esta seção descreve brevemente os algoritmos propostos em [Oliveira 2018]. Esses algoritmos visam a redução do bloqueio de requisições para estabelecimento de conexão e o uso efetivo dos recursos da redes. Os dezesseis algoritmos propostos avaliam a utilização de diferentes técnicas de proteção em redes que empregam um ou mais mecanismos de modulação adaptativa, agregação de tráfego, sobreposição de espectro e roteamento multicaminho. As métricas como bloqueio de demandas, consumo energético, fragmentação de espectro e consumo de recursos são avaliadas na provisão de proteção para EON-SDM. Todos os algoritmos apresentados garantem $100 \%$ de proteção para todas as requisições em caso de uma única falha simultâneas. Adicionalmente, o algoritmo SSSPF garante $100 \%$ de proteção contra duas falhas.

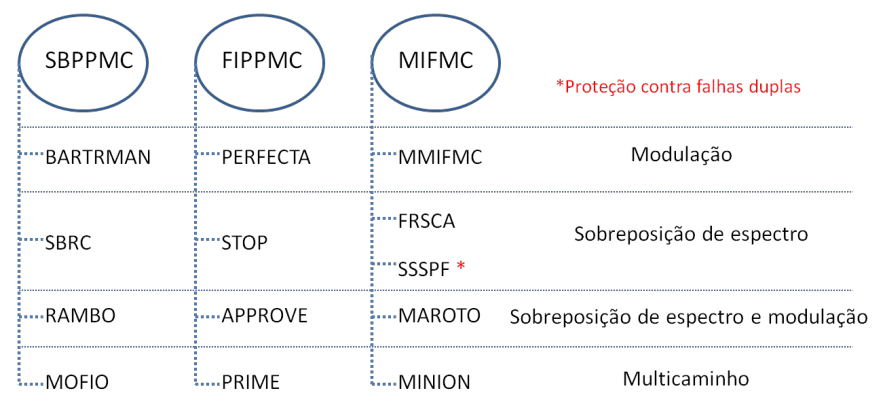

Figura 1. Visão geral das características dos algoritmos desenvolvidos

A Figura 1 apresenta uma categorização dos algoritmos desenvolvidos. Os algoritmos SBPPMC, FIPPMC e MIFMC são os algoritmos "base". Suas extensões para redes com diferentes mecanismos encontram-se denotados abaixo desses na figura 1 . Os algoritmos bases diferem pelos diferentes tipos de proteção utilizadas. Utilizam o formato de modulação fixo BPSK, que realiza a transmissão de 1 bit por símbolo. O algoritmo SBPPMC utiliza caminho de backup compartilhado (SBPP), os algoritmos FIPPMC e MIFMC utilizam p-cycle FIPP (caminho de proteção independente de falhas) e diferem pela utilização de roteamento com interferência mínima na alocação do caminho de backup. O SBPP é uma técnica de proteção que define caminhos de backup pré-planejados para caminhos primários disjuntos. O p-cycle FIPP é um esquema de proteção em que a capacidade reservada forma estruturas em anel para proteção de redes em malha. Enquanto $p$-cycle FIPP requer caminhos pré-conectados, o SBPP adota apenas caminhos pré-planejados. Em um evento de falha, um esquema SBPP precisa estabelecer dinamicamente o caminho de backup enquanto, para um p-cycle FIPP, o caminho de backup já 
está estabelecido. Os algoritmos de proteção levam a uma rápida saturação dos enlaces de rede. $\mathrm{O}$ algoritmo MIFMC, que emprega roteamento com interferência mínima, promove utilização equilibrada dos recursos, reduzindo a rejeição de requisições futuras.

Os algoritmos bases foram estendidos para os algoritmos BARTRMAN, PERFECTA e MMIFMC, com a adoção de modulação adaptativa, o que permite uso mais eficiente do espectro. Nestes algoritmos, a modulação é escolhida como uma função do comprimento dos caminhos para que a menor fração de espectro seja utilizada, e o sinal recebido seja decodificado com sucesso no destinatário. Na Tese, os formatos de modulação 64QAM, 32QAM, 16QAM, 8QAM, QPSK e BPSK foram empregados para distâncias de $125,250,500,1000,2000$ e $4000 \mathrm{~km}$, respectivamente, com slots de capacidade 75, 62,5, 50, 37,5, 25 e 12,5 Gb/s. Avaliou-se o impacto da introdução de modulação adaptativa na redução da utilização de recursos. Além disso, foi possível avaliar o comportamento das diferentes técnicas de proteção com modulação adaptativa.

Oferecer $100 \%$ de proteção exige reserva de uma grande quantidade de recursos, o que causa desperdício de capacidade e impossibilita a alocação de um maior número de conexões. Para contrapor esse alto consumo de recursos demandados para fornecer proteção, foram propostos os algoritmos SBRC, STOP, FRSCA e SSSPF. Nesses algoritmos, caso duas conexões possuírem caminhos primários disjuntos, e seus caminhos de backup percorrerem dois caminhos ópticos adjacentes em uma fibra, os dois caminhos ópticos de backup poderão sobrepor o espectro, reduzindo os recursos reservados para proteção. Além disso, através da técnica de agregação de tráfego, esses algoritmos combinam múltiplas conexões em um caminho óptico sem necessidade de bandas de guarda. A combinação de agregação de tráfego e sobreposição de espectro permite um aumento significativo na utilização do espectro. Foi possível, também, avaliar a efetividade da utilização de sobreposição de espectro para diferentes tipos de proteção. Diferentemente dos algoritmos SBRC, STOP e FRSCA, o algoritmo SSSPF utiliza $p$-cycle FIPP e sobreposição de espectro para prover proteção contra duas falhas simultâneas de enlace. A redução na utilização de recursos de backup através da sobreposição de espectro possibilitou a aceitação de um maior número de requisições, como consequência da maior disponibilidade de recursos.

Os algoritmos RAMBO, APPROVE e MAROTO utilizam modulação adaptativa e sobreposição de espectro. O uso de modulação adaptativa em conjunto com a sobreposição de espectro, tornou possível a diminuição do espectro utilizado. Enquanto a modulação adaptativa possibilita o envio de uma maior quantidade de bits por símbolos, a sobreposição de espectro reduz a utilização de recursos nos caminhos de proteção.

Com o estabelecimento e encerramento de conexões, o espectro tende a ficar fragmentado, o que diminui a possibilidade da sua utilização, aumentando, consequentemente, o bloqueio de conexões. A fim de mitigar o impacto da fragmentação do espectro, o roteamento multicaminho foi utilizado nos algoritmos MOFIO, PRIME e MINION.

\section{Resultados Obtidos}

Para avaliar o desempenho dos algoritmos proposto na Tese [Oliveira 2018], experimentos de simulação foram realizados utilizando o simulador FlexGridSim [Moura e Drummond]. Em cada simulação, 100.000 requisições foram geradas. Utilizou-se o método de replicação independente e adotou-se nível de confiança de $95 \%$ para os intervalos de confiança. Diferentes topologias com diferentes conectividades foram empregadas nas simulações: a Pan-Europan com 28 nós e 39 enlaces bidirecionais; 
a USA com 24 nós e 43 enlaces bidirecionais; e a NSF com 14 nós e 18 enlaces bidirecionais. O espectro foi dividido em 320 slots de largura de banda de $12.5 \mathrm{GHz}$ cada e as fibras simuladas possuem 7 núcleos. Variou-se a carga entre 25 e 500 erlangs em intervalos de 25 erlangs. Sete tipos de requisições foram utilizadas com demandas de banda de 25 Gbps, 50 Gbps, 125 Gbps, 200 Gbps, 500 Gbps, 750 Gbps e 1 Tbps. Todos os algoritmos propostos em [Oliveira 2018] consideram o crosstalk entre núcleos na alocação de caminhos, além disso, um caminho óptico só é estabelecido se o crosstalk gerado estiver abaixo de um nível aceitável.

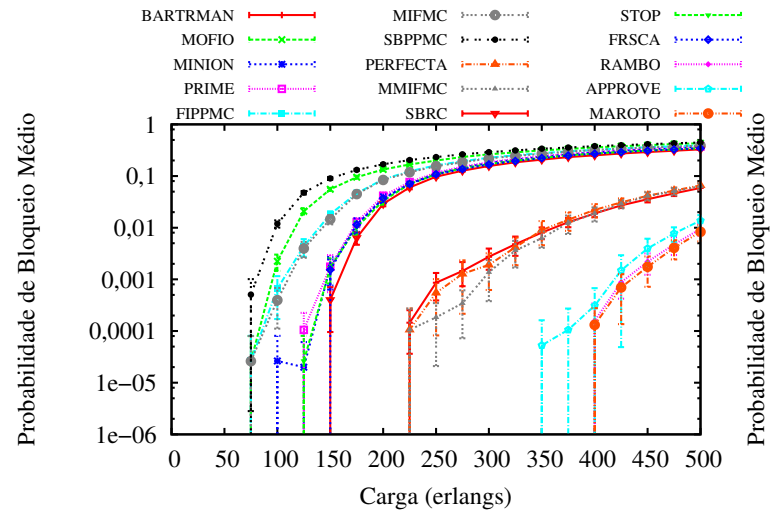

(a) Pan-European

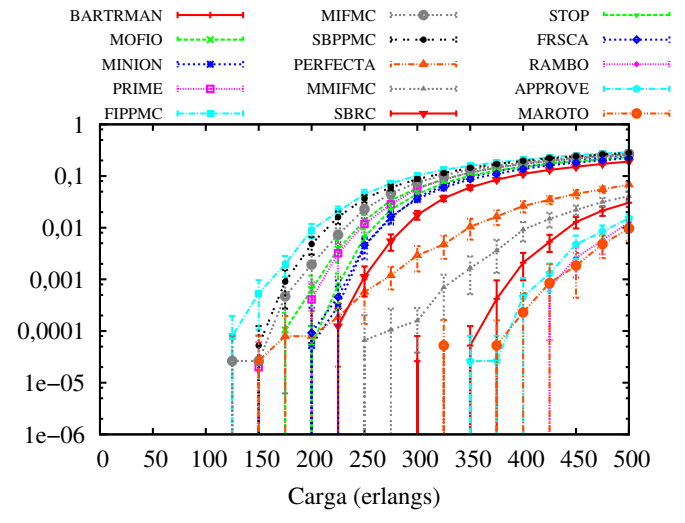

(b) USA

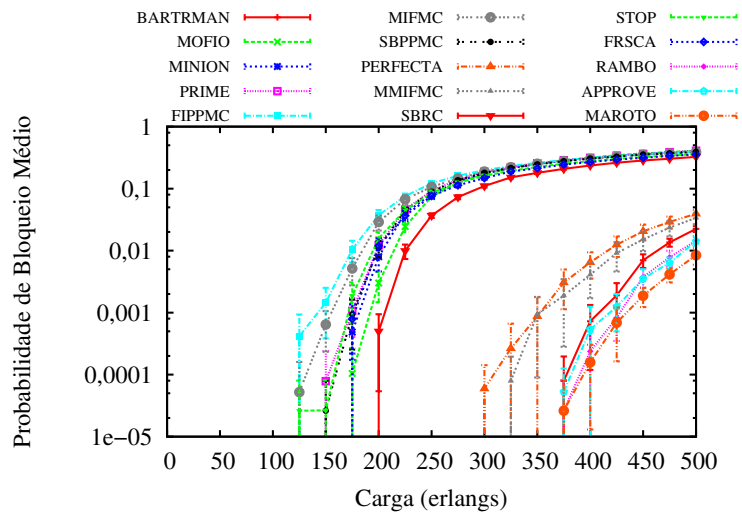

(c) NSF

Figura 2. Bloqueio de banda em função da carga da rede

As Figuras 2(a), 2(b) e 2(c) mostra a probabilidade de bloqueio (Bandwidth Blocking Ratio - BBR) para as topologias Pan-European, USA e NSF, respectivamente. Os algoritmos FIPPMC e MIFMC, que utilizam $p$-cycle, são mais indicado para redes com nós com maior conectividade, uma vez que para essas redes o uso de $p$-cycle FIPP produz valores baixos de BBR. Por sua vez, o algoritmo SBPPMC, que utiliza caminho de backup compartilhado, é mais indicado para redes com nós com menor conectividade. Os algoritmos BARTRMAN, PERFECTA, MMIFMC, RAMBO, APPROVE e MAROTO, que utilizam modulação adaptativa, produzem os menores valores de BBR para todas as topologias, quando comparado com os demais algoritmos. Isto ocorre pois estes algoritmos podem ajustar o formato de modulação de acordo com os requisitos de cada demanda, possibilitando uma alocação de recursos melhor distribuída. Dentre eles, o algoritmo MAROTO, que utiliza $p$-cycle e roteamento com interferência mínima em redes que empregam sobreposição de espectro e modulação adaptativa mostrou-se o mais indicado para todas as topologias, dado sua melhor alocação dos recursos de backup. Os algorit- 
mos SBRC, STOP, FRSCA, RAMBO, APPROVE e MAROTO reduzem a sobrecarga do espectro causada por reserva de recursos não utilizados e, assim, melhoram a eficiência espectral das redes. A sobreposição de espectro em conjunto com a modulação adaptativa mostra-se uma solução promissora pois aloca eficientemente os recursos da rede. Os algoritmos PRIME, MOFIO e MINION, que utilizam roteamento multicaminho, aumentam a possibilidade de utilização e estabelecimento de caminhos ópticos através de múltiplas rotas e, consequentemente, aumentam as chances de encontrar recursos disponíveis para suprir a banda passante demandada pelas requisições de conexão quando comparado com os algoritmos SBPPMC. FIPPMC e MIFMC, produzindo, portanto, valores menores de BBR.

Em [Oliveira 2018], outras métricas foram avaliadas. Em geral, a fragmentação apresentou grande influência no valor da BBR gerada. Os algoritmos que produziram maior BBR tenderam a produzir a maior eficiência energética pois apenas caminhos curtos foram estabelecidos. O emprego de modulação adaptativa nos algoritmos produziu uma maior eficiência energética, pois a utilização de modulação fixa, subutiliza o espectro realizando maior consumo energético. Os algoritmos que utilizam agregação de tráfego e sobreposição de espectro reduziram a fragmentação e a BBR produzida por estes algoritmos. Apesar de utilizar agregação de tráfego e sobreposição de espectro, o crosstalk per slot $(\mathrm{CpS})$ produzido por esses algoritmos é alto, devido à alta utilização do espectro. Com o emprego conjunto das técnicas de modulação adaptativa, agregação de tráfego e sobreposição de espectro houve a diminuição do bloqueio das requisições. A aceitação de um maior número de requisições gera consequentemente valores de $\mathrm{CpS}$ maiores. Os resultados mostraram que algoritmos que utilizam roteamento multicaminho conseguem prover uma maior eficiência energética, devido ao balanceamento de recursos utilizados na rede.

\section{Trabalhos Publicados}

A Tabela 1 lista os artigos produzidos a partir dos resultados da Tese [Oliveira 2018].

\section{Conclusões}

Neste artigo, foram apresentadas as contribuições da tese de doutorado [Oliveira 2018]. Um amplo estudo sobre proteção em redes ópticas elásticas com multiplexação espacial foi realizado. Os algoritmos desenvolvidos foram avaliados para diferentes topologias, métricas e cargas da rede. Os algoritmos foram propostos para redes que empregam diferentes mecanismos, tais como: agregação de tráfego, sobreposição de espectro e modulação adaptativa. Os algoritmos propostos aumentam a aceitação de requisições para estabelecimento de conexões e levam em consideração o crosstalk existente na rede. Além disso, reduzem a fragmentação dos enlaces da rede, o consumo energético, e o número de saltos do caminho primário e de backup. Os resultados obtidos são originais em tópico muito pouco explorado na literatura. Espera-se que as contribuições da Tese possam propiciar uma Internet que transporte volume de dados muito maiores que os atuais com proteção a falhas.

\section{Referências}

Fujii, S., Hirota, Y., Tode, H., e Watanabe, T. (2017). On-demand routing and spectrum allocation for energy-efficient aod nodes in sdm-eons. IEEE/OSA Journal of Optical Communications and Networking, 9(11):960-973.

Moura, P. M. e Drummond, A. C. FlexGridSim: Flexible Grid Optical Network Simulator. http://www.Irc.ic.unicamp.br/FlexGridSim/. 
Tabela 1. Trabalhados publicados como resultados da Tese

\begin{tabular}{llll}
\hline Referência & Periódico & Qualis & Situação \\
\hline [Oliveira e Fonseca 2019b] & - & - & A submeter \\
[Oliveira e Fonseca 2019e] & Optical Switching and Networking & B1 & Submetido \\
[Oliveira e Fonseca 2019a] & Journal of Network and Computer Applications & A2 & Publicado \\
[Oliveira e Fonseca 2018b] & IEEE Communications Letters & A1 & Publicado \\
[Oliveira e Fonseca 2017b] & IEEE Latin America Transactions & B4 & Publicado \\
\hline Referência & Conferência & Qualis & Situação \\
\hline [Oliveira e Fonseca 2019c] & IEEE Globecom 2019 & A1 & Submetido \\
[Oliveira e Fonseca 2019g] & SBRC 2019 & B2 & Aceito \\
[Oliveira e Fonseca 2019d] & IEEE ICC 2019 & A1 & Aceito \\
[Oliveira e Fonseca 2019f] & IEEE ICC 2019 & A1 & Aceito \\
[Oliveira e Fonseca 2018c] & IEEE ICC 2018 & A1 & Publicado \\
[Oliveira e Fonseca 2018a] & SBRC 2018 & B2 & Publicado \\
[Oliveira e Fonseca 2017d] & IEEE Globecom 2017 & A1 & Publicado \\
[Oliveira e Fonseca 2017c] & SBRC 2017 & B2 & Publicado \\
[Oliveira e Fonseca 2017e] & IEEE Latincom 2017 & B2 & Publicado \\
[Oliveira e Fonseca 2017a] & IEEE ICC 2017 & A1 & Publicado \\
[Oliveira e Fonseca 2016a] & IEEE Globecom 2016 & A1 & Publicado \\
[Oliveira e Fonseca 2016b] & IEEE Latincom 2016 & B2 & Publicado \\
[Oliveira e Fonseca 2016d] & IEEE Latincom 2016 & B2 & Publicado \\
[Oliveira e Fonseca 2016c] & SBRC 2016 & B2 & Publicado \\
\hline
\end{tabular}

Oliveira, H. M. e Fonseca, N. L. (2019a). Routing, spectrum and core assignment algorithms for protection of space division multiplexing elastic optical networks. Journal of Network and Computer Applications, 128:78 - 89.

Oliveira, H. M. N. S. (2018). Proteção em Redes Ópticas Elásticas com Multiplexação Espacial. PhD thesis, Universidade Estadual de Campinas.

Oliveira, H. M. N. S. e Fonseca, N. L. S. (2016a). Algorithm for protection of space division multiplexing elastic optical networks. In IEEE Globecom, pages 1-6.

Oliveira, H. M. N. S. e Fonseca, N. L. S. (2016b). The minimum interference p-cycle algorithm for protection of space division multiplexing elastic optical networks. In IEEE Latincom, pages 1-6.

Oliveira, H. M. N. S. e Fonseca, N. L. S. (2016c). Proteçao de redes opticas elásticas baseada em agregaç ao de tráfego, sobreposiç ao de espectro e p-cycle fipp. In SBRC.

Oliveira, H. M. N. S. e Fonseca, N. L. S. (2016d). Traffic grooming and spectrum overlap in fipp p-cycle for protection of elastic optical networks. In IEEE Latincom, pages 1-6.

Oliveira, H. M. N. S. e Fonseca, N. L. S. (2017a). Algorithm for shared path for protection of space division multiplexing elastic optical networks. In IEEE ICC, pages 1-6.

Oliveira, H. M. N. S. e Fonseca, N. L. S. (2017b). The minimum interference p-cycle algorithm for protection of space division multiplexing elastic optical networks. IEEE Latin America Transactions, 15(7):1342-1348.

Oliveira, H. M. N. S. e Fonseca, N. L. S. (2017c). Proteção de redes ópticas elá sticas com multiplexação espacial baseada em modulação, p-cycle fipp e interferência mínima. In SBRC, volume 35 .

Oliveira, H. M. N. S. e Fonseca, N. L. S. (2017d). Routing, spectrum, core and modulation level assignment algorithm for protected sdm optical networks. In IEEE Globecom, 
pages $1-6$.

Oliveira, H. M. N. S. e Fonseca, N. L. S. (2017e). Sharing spectrum and straddling p-cycle fipp for protection against two simultaneous failures in sdm elastic optical networks. In IEEE Latincom, pages 1-6.

Oliveira, H. M. N. S. e Fonseca, N. L. S. (2018a). Proteção de redes ópticas elásticas com multiplexação espacial baseada em agregação de tráfego, sobreposição de espectro e compartilhamento de caminho de backup. In $S B R C$, volume 36.

Oliveira, H. M. N. S. e Fonseca, N. L. S. (2018b). Protection, routing, modulation, core, and spectrum allocation in sdm elastic optical networks. IEEE Communications Letters, 22(9):1806-1809.

Oliveira, H. M. N. S. e Fonseca, N. L. S. (2018c). Spectrum overlap and traffic grooming in p-cycle algorithm protected sdm optical networks. In IEEE ICC, pages 1-6.

Oliveira, H. M. N. S. e Fonseca, N. L. S. (2019b). Backup, routing, spectrum and core allocation in eons-sdm for efficient spectrum utilization. -. (À ser submetido).

Oliveira, H. M. N. S. e Fonseca, N. L. S. (2019c). Multipath routing, spectrum and core allocation in protected SDM elastic optical networks. In 2019 IEEE Global Communications Conference: Communication QoS, Reliability and Modeling (IEEE Globecom 2019 CQRM), Waikoloa, USA.

Oliveira, H. M. N. S. e Fonseca, N. L. S. (2019d). P-cycle protected multipath routing, spectrum and core allocation in sdm elastic optical networks. In IEEE ICC, pages 1-6. (Submetido).

Oliveira, H. M. N. S. e Fonseca, N. L. S. (2019e). Protection in elastic optical networks using failure-independent path protecting p-cycles. Optical Switching and Networking. (Submetido).

Oliveira, H. M. N. S. e Fonseca, N. L. S. (2019f). Protection, routing, spectrum and core allocation in eons-sdm for efficient spectrum utilization. In IEEE ICC, pages 1-6. (Submetido).

Oliveira, H. M. N. S. e Fonseca, N. L. S. (2019g). Proteção e roteamento multicaminho em redes ópticas elásticas com multiplexação por divisão espacial. In $S B R C$, volume 36. (Submetido).

Tan, Y., Zhu, R., Yang, H., Zhao, Y., Zhang, J., Liu, Z., Qu, Q., e Zhou, Z. (2016). Crosstalk-aware provisioning strategy with dedicated path protection for elastic multicore fiber networks. In International Conf. on Optical Communications and Networks, pages $1-3$.

Tode, H. e Hirota, Y. (2017). Routing, spectrum, and core and/or mode assignment on space-division multiplexing optical networks [invited]. IEEE/OSA Journal of Optical Communications and Networking, 9(1):A99-A113.

Yang, M., Zhang, Y., e Wu, Q. (2018). Routing, spectrum, and core assignment in sdmeons with mcf: node-arc ilp/milp methods and an efficient xt-aware heuristic algorithm. IEEE/OSA Journal of Optical Communications and Networking, 10(3):195-208.

Yin, S., Huang, S., Guo, B., Li, X., Wang, C., e Huang, H. (2017). Inter-core crosstalk aware routing, spectrum and core allocation in multi-dimensional optical networks. In 2017 Opto-Electronics and Communications Conf. (OECC) and Photonics Global Conf. $(P G C)$, pages $1-4$. 